\title{
Cultura tributaria para el incremento de los ingresos a los comerciantes del mercado modelo, Chachapoyas, 2018
}

\section{Tax culture for the increase of income to the traders of the model market, Chachapoyas, 2018}

Carlos Alberto Hinojosa Salazar ${ }^{1}$

\section{RESUMEN}

La presente investigación tuvo por objetivo determinar como la cultura tributaria incrementará los ingresos a los comerciantes del mercado modelo de la ciudad de Chachapoyas, 2018. Para el desarrollo de la investigación se hizo uso del método descriptivo que permitió describir las variables de estudio. La muestra de estudio estuvo conformada por 149 comerciantes de la ciudad de Chachapoyas. El muestreo aplicado fue no probabilística intencional. Para la recolección de datos se utilizó la técnica de la encuesta. En los resultados se muestra que para los comerciantes del Mercado Modelo de la ciudad de Chachapoyas los tributos no contribuyen a mejorar la calidad de vida de la población, percepción causada por la falta de conocimiento sobre cultura tributaria; lo que limita su desarrollo económico y social. Concluyendo que los comerciantes del Mercado Modelo de la ciudad de Chachapoyas tienen un nivel de conocimiento bajo en el tema de tributación; este bajo conocimiento generalmente se debe a la informalidad de las actividades de los pequeños empresarios.

Palabras clave: Contribuyente, Cultura Tributaria, Ingresos, Mercado de Abastos

\begin{abstract}
The objective of this research was to determine how the tax culture will increase the income of merchants in the model market of the city of Chachapoyas, 2018. For the development of the research, the descriptive method was used to describe the study variables. The study sample consisted of 149 merchants from the city of Chachapoyas. The sampling applied was intentional non-probabilistic. For data collection, the survey technique was used. The results show that for the traders of the Model Market of the city of Chachapoyas, taxes do not contribute to improving the quality of life of the population, perception caused by the lack of knowledge about tax culture; what limits its economic and social development. Concluding that the traders of the Model Market of the city of Chachapoyas have a low level of knowledge in the subject of taxation; this low knowledge is usually due to the informality of the activities of small entrepreneurs.
\end{abstract}

Keywords: Taxpayer, Tax Culture, Income, Market Supply

Contador Público Colegiado. Docente Asociado de la Facultad de Ciencias Económicas y Administrativas de la Universidad Nacional Toribio Rodríguez de Mendoza de Amazonas; Doctor en administración; Magister en ciencias económicas: Mención finanzas por la Universidad Nacional de Trujillo.Correo electrónico: carlos.hinojosa@untrm.edu.pe 


\section{INTRODUCCIÓN}

El Estado peruano para cubrir las necesidades públicas colectivas (institucionales, sociales y políticas), necesitan disponer de recursos. Esos recursos que vienen de la sociedad son entregados al estado en calidad de administrador quien obteniéndolos se encarga de distribuir a todos los departamentos del país en sus diferentes sectores. Estos recursos que la sociedad peruana aporta a las cajas fiscales, retornan a ella en forma de servicios y obras públicas, teniendo en cuenta esto, los ciudadanos debemos proteger la provisión de los recursos a la comunidad dado que al hacerlo protegemos nuestros propios intereses. Lo que se quiere hacer es que las personas conozcan las normas tributarias, sus obligaciones y procedimientos de pago de los tributos cumpliendo voluntariamente con sus obligaciones al Estado. Estos recursos que la sociedad peruana aporta a las cajas fiscales, retornan a ella en forma de servicios y obras públicas, teniendo en cuenta esto, los ciudadanos debemos proteger la provisión de los recursos a la comunidad dado que al hacerlo protegemos nuestros propios intereses.

Según, Martínez, M. (2016), muchos comerciantes no están preparados para cumplir su rol ante la sociedad, esto es debido a la falta de cultura tributaria, sumada a la carencia de conciencia tributaria y la aplicación de valores que nacen de su propia identidad y formación, para esto es necesario ir mucho más allá y profundizar la relación que debe existir entre el estado y los ciudadanos, para ello es necesario desarrollar una cultura tributaria que permita a los comerciantes concebir las obligaciones tributarias como un deber sustantivo acorde con los valores democráticos.

Para que el Perú pase al nivel de una economía desarrollada requiere una mejora de la calidad de sus políticas públicas y mejores instituciones. Chile, a pesar que su economía tiene un importante nivel primario, se viene acercando al nivel de las economías desarrolladas, justamente porque viene mejorando sus políticas públicas y sus instituciones. Por esta razón el 2012 Chile registró los más altos niveles de PBI per cápita de la región sudamericana. Según estudios realizados por la Superintendencia Nacional de Administración Tributaria (SUNAT), el Perú es la quinta economía más informal del mundo, pues alrededor del $60.9 \%$ de nuestra economía existe al margen de la ley, el estado pierde anualmente $\mathrm{S} /$ 1,000.00 millones entre impuesto a la renta $\mathrm{e}$ impuesto general a las ventas de unos 62,000 contribuyentes.
En la ciudad de Chachapoyas, los últimos 20 años se ha visto con un crecimiento vertiginoso por cuanto la Universidad Nacional Toribio Rodríguez de Mendoza de Amazonas, ha traído consigo la aparición de nuevos negocios que formal o informal pueden tributar o no, es así como el mercado de abastos que por los años de 1991 solo contaba con una planta o un solo piso, actualmente tiene dos pisos y aun así ha quedado pequeño por la cantidad de usuarios, es así como muchos de estos comerciantes por la coyuntura existente de ubicarse en el mercado de abastos no pagan los impuestos respectivos debido mayormente a un desconocimiento de las normas tributarias, es por esta razón que con la presente investigación se pretende buscar alternativas y plantear una alternativa de cultura tributaria y de esta manera incrementar los ingresos tributarios lo cual redundará en mayores beneficios para nuestra población, el cumplimiento voluntario de las obligaciones tributarias del Estado.

\section{MATERIAL Y MÉTODOS}

El diseño de investigación utilizado fue descriptiva. Muestra

M1: Se tomó en su totalidad a 149 comerciantes de la ciudad de Chachapoyas.

\section{Métodos y técnicas}

Para el desarrollo de la investigación se hizo uso del método descriptivo que permitió describir las variables de estudio.

Para la recolección de datos se utilizó la técnica de la encuesta.

\section{RESULTADOS}

\subsection{Resultados de la encuesta aplicada a los comerciantes de la ciudad de Chachapoyas}

Tabla $N^{\circ} 1: \_$Usted considera que pagar sus tributos ayudan a mejorar la calidad de vida de los pobladores?

\begin{tabular}{|l|l|l|l|}
\hline & $\mathbf{f i}$ & hi & HI \\
\hline Si & 64 & $43 \%$ & $43 \%$ \\
\hline No & 85 & $57 \%$ & $100 \%$ \\
\hline Total & 149 & $100 \%$ & \\
\hline
\end{tabular}

Fuente: Encuesta

En la figura 01 , se muestra que el $57 \%$ consideran que pagar sus tributos ayudan a mejorar la calidad de vida de los pobladores y el $43 \%$ consideran que no- 


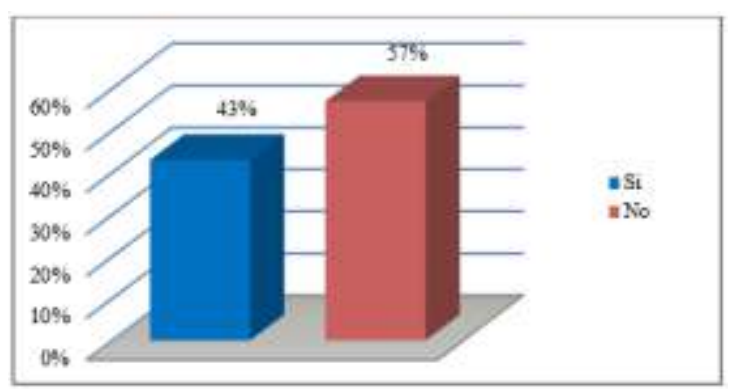

Figura $\mathrm{N}^{\circ}$ 1: ¿Usted considera que pagar sus tributos ayudan a mejorar la calidad de vida de los pobladores?

Tabla N²: Tabla de contingencia ¿Conoces los tributos que como comerciante está obligado a cumplir? / Mencione cuales son.

\begin{tabular}{|c|c|c|c|c|}
\hline \multirow{3}{*}{$\begin{array}{l}\text { Mencione } \\
\text { cuales son y } \\
\text { para qué } \\
\text { sirven }\end{array}$} & \multicolumn{4}{|c|}{$\begin{array}{l}\text { ¿Conoces los tributos que como } \\
\text { comerciante está obligado a } \\
\text { cumplir? }\end{array}$} \\
\hline & \multicolumn{3}{|c|}{$\mathrm{Si}$} & No \\
\hline & fi & $\%$ & fi & $\%$ \\
\hline $\begin{array}{l}\text { Alquiler del } \\
\text { puesto }\end{array}$ & 124 & $83.2 \%$ & 0 & $0 \%$ \\
\hline $\begin{array}{l}\text { Alquiler del } \\
\text { puesto - } \\
\text { RUS }\end{array}$ & 1 & $0.7 \%$ & 0 & $0 \%$ \\
\hline No saben & 0 & $0 \%$ & 24 & $16.1 \%$ \\
\hline Total & 125 & $83.9 \%$ & 24 & $16.1 \%$ \\
\hline
\end{tabular}

Fuente: Encuesta

En la figura 02 , se muestra que el $83.2 \%$ indico que el tributo es el alquiler del puesto y el $0.7 \%$ indico que el tributo es el RUS; el $16.1 \%$ indico no conocer los tributos.

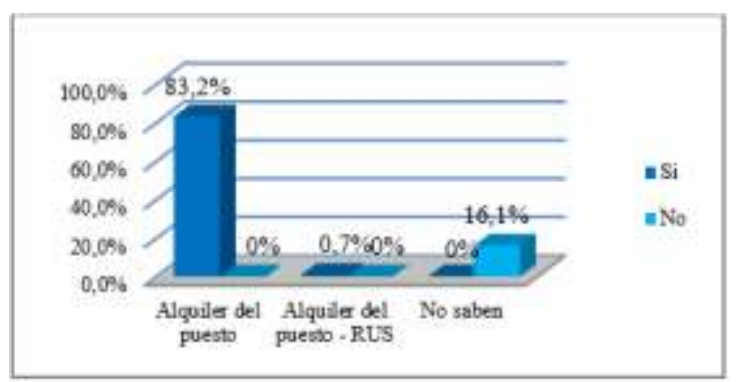

Figura $\mathrm{N}^{\circ} 02$ : Figura agrupada ¿Conoces los tributos que como comerciante está obligado a cumplir? / Mencione cuales son.

Tabla $N^{\circ}$ 3: Tabla de contingencia Sexo ¿Conoce usted sus derechos y obligaciones tributarias como contribuyente?

\begin{tabular}{lllll} 
& \multicolumn{4}{l}{$\begin{array}{l}\text { ¿Conoce usted sus derechos y } \\
\text { obligaciones tributarias como } \\
\text { contribuyente? }\end{array}$} \\
\hline Sexo & \multicolumn{3}{c}{$\mathrm{Si}$} & \multicolumn{2}{c}{ No } \\
\cline { 2 - 5 } fi & $\%$ & fi & $\%$ \\
Femenino & 64 & $43.0 \%$ & 37 & $24.8 \%$ \\
Masculino & 25 & $16.8 \%$ & 23 & $15.4 \%$ \\
\hline
\end{tabular}

En la figura 04, se muestra que del grupo femenino el $43 \%$ indico que si conoce y el $24.8 \%$ indico que no conoce, del grupo masculino el $16.8 \%$ indico que si conoce y el $15.4 \%$ indico que no conoce.



Figura $N^{\circ}$ 03: Tabla de contingencia Sexo * ¿Conoce usted sus derechos y obligaciones tributarias como contribuyente?

\section{DISCUSIÓN}

En la Tabla/Figura 01: según la investigación realizada se ha obtenido que del $100 \%$ de las personas encuestadas, según la interrogante $¿$ Usted considera que pagar sus tributos ayudan a mejorar la calidad de vida de los pobladores?, el $43 \%$ considera que los tributos contribuyen a mejorar la calidad de vida de la población y el $57 \%$ considera que los tributos no contribuyen a mejorar la calidad de vida de la población.

En su estudio Rivera y Silvera encontraron que la falta de un conocimiento serio y sostenible del proceso de formalización tributaria entre los comerciantes informales ubicados en la zona central del Cantón Milagro interrumpe su desarrollo económico y social. Los resultados obtenidos cuyos análisis señalan inexistencia de tramites tributarios, fomento de comerciantes informales en la vía pública y carencia de cursos de capacitación en materia administrativa y tributaria.

Comparando ambos estudios se encuentra una similitud; ya que, en la investigación realizada por Rivera y Silvera se encontró que la falta de un conocimiento serio y sostenible de formalización tributaria interrumpe su desarrollo económico y social, y en el presente estudio la mayoría de personas encuestadas indicaron que los tributos no contribuyen a mejorar la calidad de vida de la población. En los dos casos el principal problema es la falta de conocimiento en temar relacionados a la tributación.

En la actualidad podemos apreciar que tanto la cultura y la conciencia tributaria son factores muy importantes en la población; debido a que ambos conceptos ayuda a que podamos comprender que los 
tributos son recursos que recauda el Estado para ser administrados a favor del bienestar de la población en su conjunto; es decir, estos recursos serán devueltos prestando servicios públicos de calidad.

En la Tabla/Figura 02: según la investigación realizada se ha obtenido que del $100 \%$ de las personas encuestadas, de acuerdo a si conocen los tributos y cuáles son, del grupo que indico que si conocía los tributos el $83.2 \%$ indico que el tributo es el alquiler del puesto y el $0.7 \%$ indico que el tributo es el RUS; el 16.1\% indico no conocer los tributos. Cabe resaltar que la mayoría de persona que indicaron conocer los tributos lo hicieron de manera errónea ya que el pago del alquiler de un puesto no se considera tributo.

Huere, X. y Muña, A. realizaron una investigación titulada: "Cultura tributaria para la formalización de las Mypes de los mercados de la provincia de Huancayo", cuyos resultados fueron los siguientes: la gran mayoría de propietarios y/o administradores de las MYPEs encuestados, a través de sus respuestas, determinaron que existe un nivel bajo de conocimiento sobre tributos y ello repercute en la formalización de las mismas.

Comparando los resultados de la presente investigación con los resultados obtenidos en la investigación realizada por Huere, X. y Muña, A. se encuentra una similitud; debido a que en ambos estudios los encuestados presentaron un nivel de conocimiento bajo en el tema de tributación; este bajo conocimiento generalmente se debe a la informalidad de las actividades de los pequeños empresarios.

Los ciudadanos deben poseer un ato conocimiento sobre temas relacionados a la tributación; la falta de conocimiento genera evasión tributaria, ocasionando un retraso en el desarrollo económico y social del país. El estado tiene como principal función velar por el bienestar común y brindar servicios básicos de calidad, para lo cual necesita recaudar recursos, cuya principal fuente son los tributos pagados por los contribuyentes.

En la Tabla/Figura 03: según la investigación realizada se ha obtenido que del $100 \%$ de las personas encuestadas, de acuerdo a si conocen sus derechos y obligaciones tributarias según el sexo, del grupo femenino el $43 \%$ indico que si conoce y el $24.8 \%$ indico que no conoce, del grupo masculino el $16.8 \%$ indico que si conoce y el $15.4 \%$ indico que no conoce.

Banfi, D. desarrollo un artículo titulado: "La Cultura Tributaria para el cumplimiento de la Obligación Tributaria"; cuyos resultados indicaron que la cultura tributaria es una manifestación del comportamiento social, lo que provoca que los seres humanos actúen dentro de las normas legales establecidas, cumpliendo así con las distintas obligaciones no solo en materia tributaria sino a nivel social evitando sanciones y conductas contrarias al deber ser.

Comparando los resultados obtenidos en la presente investigación con los resultados obtenidos en la investigación Banfi, D. se encuentran diferencias; ya que en la investigación de Banfi las personas encuestadas si tenían conocimientos sobre cultura tributaria y el principal problema encontrado en temas tributarios es el comportamiento social (valores), mientras que en la presente investigación el principal problema es el nivel bajo de conocimiento que presentan los pequeños empresarios en temas tributarios.

La relación Ciudadano - Estado es un tema muy importante para abordar el tema tributario; debido a que su estudio involucra múltiples dimensiones, en el tema tributario podemos hablar del nivel de conocimiento y la escala de valores de los ciudadanos. Al fortalecer tanto el nivel de conocimiento cono una escala valores se llega a una verdadera cultura en materia de tributos y así poner en práctica de una forma consciente, responsable, transparente y ético el cumplimiento voluntario de la obligación tributaria.

\section{CONCLUSIONES}

1. La cultura tributaria incrementa el ingreso de los comerciantes, ya que un comerciante formal tiene más oportunidades de incrementar su cartera de clientes a través de convenios con instituciones Publio - privadas por la confianza que genera al emitir comprobantes de pago.

2. Para los comerciantes del Mercado Modelo de la ciudad de Chachapoyas los tributos no contribuyen a mejorar la calidad de vida de la población, percepción causada por la falta de conocimiento sobre cultura tributaria; lo que limita su desarrollo económico y social.

3. Los comerciantes del Mercado Modelo de la ciudad de Chachapoyas tienen un nivel de conocimiento bajo en el tema de tributación; este bajo conocimiento generalmente se debe a la informalidad de las actividades de los pequeños empresarios.

4. La falta de conocimiento genera evasión tributaria, ocasionando un retraso en el desarrollo económico y social del país. El estado tiene como principal función velar por el bienestar común y brindar servicios básicos de calidad, para lo cual 
necesita recaudar recursos, cuya principal fuente son los tributos pagados por los contribuyentes.

\section{REFERENCIAS BIBLIOGRÁFICAS}

Banfi, D. (2013). La Cultura Tributaria para el cumplimiento de la Obligación Tributaria. Venezuela: URBE - Universidad Privada Dr. Rafael Belloso Chacín.

Huera, X. у. (2016). “Cultura tributaria para la formalización de las Mypes de los mercados de la provincia de Huancayo",. (U. N. Peru, Ed.) Facultad de Contabilidad.

Martinez, M. (2016.). La cultura tributaria y su impacto en el cumplimiento de las obligaciones tributarias mensuales de igvrenta en los comerciantes minoristas de abarrotes del mercado modelo de Sullana en el año 2015. Piura - Perú. : Universidad Catolica Los Angeles Chimbote.

Rivera, J., \& Silvera, I. (2012). Formalizacion tributaria de los comerciantes informales en la zona central del Canton Milagro. Milagro Ecuador: Universidad Estatal de Milagro.

Salas, O. (2014). ESTRATEGIAS GERENCIALES SOBRE LA CULTURA TRIBUTARIA. $\mathrm{O}$ b t e n i d o d e http://www.geocities.ws/onelysalasp/fase 1/t rabajo4.html 